\title{
Quantum localization issues in nonlinear frequency conversion and harmonic generation
}

\author{
Kayn A. Forbes, Jack S. Ford and David L. Andrews* \\ School of Chemistry, University of East Anglia, Norwich NR4 7TJ, United Kingdom
}

\begin{abstract}
Issues of a fundamental quantum origin exert a significant effect on the output mode structures in optically parametric processes. An assumption that each frequency conversion event occurs in an infinitesimal volume produces uncertainty in the output wave-vector, but a rigorous, photon-based theory can provide for a finite conversion volume. It identifies the electrodynamic mechanisms operating within the corresponding region of space and time, on an optical wavelength and cycle timescale. Based on quantum electrodynamics, this theory identifies specific material parameters that determine the extent and measure of delocalized frequency conversion, and its equations deliver information on the output mode structures. The results also indicate that a system of optimally sized nanoparticles can display a substantially enhanced efficiency of frequency conversion.

Keywords: photonics, entanglement, photon-pairs, delocalized, quantum electrodynamics, quantum optics, quantum entanglement, photon correlation, quantum information, non-linear optics, second harmonic generation.
\end{abstract}

\section{INTRODUCTION}

The nonlinear optical processes known as second harmonic generation (SHG) and spontaneous parametric down-conversion (SPDC) have both had a profound influence in optical physics since their initial discoveries. ${ }^{1-3}$ The generation of optical harmonics - which in the simplest of cases involves the conversion of two pump photons into a single double frequency output photon - has acquired applications in a large number of research areas. High resolution SHG microscopy has been shown to be a powerful tool in the clinical analysis and diagnosis of a wide range of diseases ${ }^{4-5}$, where the ability to image tissue non-invasively, using nonlinear optical microscopic techniques, has proven to be indispensable - and to yield a plethora of advantages over conventional imaging methods. ${ }^{6-8}$ Another application of optical harmonic generation involves utilizing SHG as an optical probe to characterize interfaces and surface dynamics. ${ }^{9-10}$ Contemporary work in the field has centred upon creating next-generation SHG materials ${ }^{11}$ that exhibit significantly enhanced generation of optical harmonics using semiconductor metamaterials ${ }^{12}$, exciton-enhancment ${ }^{13}$, and atomic crystals ${ }^{14}$. The process of SPDC is the time reversal of SHG (see Fig. 1) whereby a single pump photon splits into a pair of output twin photons, which again conserve energy. The output constitutes an entangled correlated photon pair15-16, a feature that itself links with another intensively researched field, ${ }^{17-18}$ with major implications in a multitude of contemporary areas, particularly in the field of quantum information. ${ }^{19-21}$

At the most significant level, both the processes of SHG and SPDC are third-order electric dipole couplings to the radiation. The creation and annihilation of photons occur in a localized fashion, in the sense that the positional uncertainty of the input and output photons is very small (a restriction imposed by the uncertainty principle itself) and therefore it is generally assumed that the output photon or photons originate from the same positional origin as where the associated input photon annihilation takes place. However, recent work has highlighted that, for each conversion, the three correlated photon events need not be always be collocated. In particular, the individual photons that constitute the correlated pairs produced by SPDC can come from separate, distinct locations in the nonlinear crystal, through a delocalized mechanism. ${ }^{22-23}$ The work presented within is 
concerned with extending the physics behind this new mechanism from a specific optical process (SPDC), and applying it to another optical phenomenon, namely SHG. Identifying the variation of significance in the delocalized mechanism also leads to results that indicate an optimum size for nanoparticles, in a medium designed for frequency conversion.

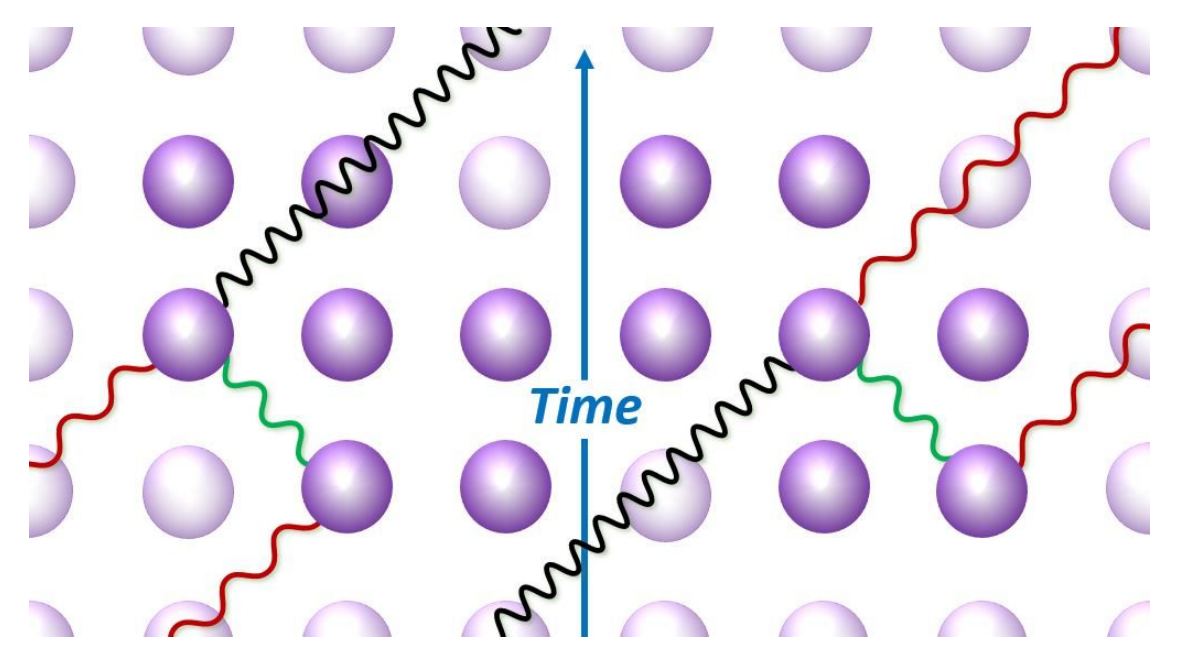

Figure 1. Depiction highlighting the time-symmetry relationship between the delocalized mechanisms of SHG (left) and SPDC (right) within a nonlinear crystal consisting of optical centers (purple spheres). Wavy lines represent photons: black photons are of wavelength $2 \lambda$; red photons are of a wavelength $\lambda$; green photons represent virtual photons.

\section{THEORETICAL BACKGROUND}

The correct description for the interaction of light and matter requires both to be quantized. ${ }^{24-25}$ The theory of quantum electrodynamics satisfies such a requirement, treating the interaction between radiation and charged particles as quantized matter-photon interactions. In non-covariant $\mathrm{QED}^{26}$ - where the material part of the dynamical system has non-relativistic energies, - the Power-Zienau-Woolley (PZW) Hamiltonian ${ }^{27-28}$ is routinely employed:

$$
H=H_{\mathrm{rad}}+\sum_{\xi}^{N} H_{\mathrm{mol}}(\xi)+\sum_{\xi}^{N} H_{\mathrm{int}}(\xi)
$$

The PZW Hamiltonian (1) is written as a sum of three terms: (i) the second-quantized Hamiltonian for the radiation field in vacuo, $H_{\text {rad }}$; (ii) a sum over all particles with corresponding nonrelativistic Hamiltonians, $H_{\text {mol }}(\xi)$; (iii) a sum of the interaction Hamiltonians that describe the coupling between each particle and the electromagnetic field, $H_{\text {int }}(\xi)$.

The last term in (1) is generally of most interest as it accounts for the coupling and exchange of energy between the radiation field and matter. This interaction Hamiltonian is generally cast in the multipolar framework $^{29}$ as it simplifies computation of physically observable rates and energies of processes and phenomena $^{30}$, respectively. The leading terms in the multipolar expansion of $H_{\text {int }}$ are:

$$
H_{\mathrm{int}}^{\text {mult }}(\xi)=\sum_{\xi}\left\{-\varepsilon_{0}^{-1} \mu_{i}(\xi) d_{i}^{\perp}\left(\boldsymbol{r}_{\xi}\right)-m_{i}(\xi) b_{i}\left(\boldsymbol{r}_{\xi}\right)-\varepsilon_{0}^{-1} Q_{i j}(\xi) \nabla_{j} d_{i}^{\perp}\left(\boldsymbol{r}_{\xi}\right)+\ldots\right\}
$$


where $\mu_{i}(\xi), m_{i}(\xi)$, and $Q_{i j}(\xi)$ are components of the electric-dipole, magnetic-dipole and electric-quadrupole tensor operators, respectively: $d_{i}^{\perp}\left(\boldsymbol{r}_{\xi}\right)$ represents components of the transverse displacement electric field operator, and $b_{i}\left(\boldsymbol{r}_{\xi}\right)$ the corresponding magnetic field operator. Keeping only the first term of equation (2) provides for calculations within the well-known electric-dipole approximation - a constraint adopted in the primary determination of non-local features in nonlinear optics. Higher order couplings become significant when studying chiral discriminatory effects in optical processes including forces ${ }^{31-33}$ and non-linear optics. ${ }^{34-35}$ A quantum field description that gives the correct results for condensed phase materials requires account to be taken of the modification of the fields experienced (and produced) by any specific optical centre(s), due to its surroundings by the other atoms and molecules in the material. In QED, this is accommodated for in the radiation field operators ${ }^{36-37}$ which, within the electric dipole approximation, can be written as;

$$
\boldsymbol{d}^{\perp}(\boldsymbol{r})=i\left(\frac{\hbar \varepsilon_{0} \omega}{2 V n^{2}}\right)^{\frac{1}{2}}\left(\frac{n+2}{3}\right)\left[\boldsymbol{e}_{\boldsymbol{k}}^{(\eta)} a_{\boldsymbol{k}}^{(\eta)} \mathrm{e}^{i \boldsymbol{k} \cdot \boldsymbol{r}}-\overline{\boldsymbol{e}}_{\boldsymbol{k}}^{(\eta)} a_{\boldsymbol{k}}^{\dagger(\eta)} \mathrm{e}^{-i \boldsymbol{k} \cdot \boldsymbol{r}}\right],
$$

The above displacement electric field operator is a modification of the fully-rigorous and general form. It is legitimate for its application here as it has been assumed the conservation of photon momentum is intrinsic to the coherent processes i.e. $\boldsymbol{k}=2 \boldsymbol{k}^{\prime}$ for SPDC (the optical frequency of the input is such that $n(\omega) \omega=c k \equiv c|\boldsymbol{k}|$, and for the output $\left.n\left(\omega^{\prime}\right) \omega^{\prime}=c\left|\boldsymbol{k}^{\prime}\right|\right)$ and $2 \boldsymbol{k}=\boldsymbol{k}^{\prime}$ for SHG. This means that for SPDC and SHG to occur at any sizeable rate in the condensed phase, the process must occur with index-matching - and for simplicity we can neglect any minor difference in refractive index at frequencies $\omega$ and $\omega^{\prime}$ the index therefore appears as simply $n$.

To describe how to correctly compute $M_{f}$, it is necessary to go over some details of the theory: the processes of SPDC and SHG are predominantly single-center processes, where it is usually assumed that correlated photon creation and annihilation events occur at the same optical center which we can label A - in shorthand we can write the leading order to these two nonlinear optical processes as [AAA] (three photonmatter interactions at position $\mathrm{A}$, engaging third order coupling in $H_{\text {int }}$ ). Beyond this specifically local leading term, it emerges that additional contributions can only be of order $3+2 p$, where $p$ is a positive integer. ${ }^{23}$ Therefore, the leading corrections come from fifth order, necessarily requiring that the two additional photon events are the creation and annihilation of a photon in an arbitrary mode - i.e. a virtual photon, fleetingly borrowing energy from the vacuum field. A variety of possibilities arises for the additional contributions, but the ones that we are interested here, due to their non-local properties, are of the type [AAABB]. This signifies a mechanism wherein SPDC and SHG are cooperatively delivered by two centers, A and B, coupled by a virtual photon created at one center and annihilated at the other (see Fig. 1). In short, it is found that for $N$ optical centers:

$$
M_{f i}=\sum_{\xi}^{N} M_{f i}^{(3)}(\xi)+\sum_{\xi}^{N} M_{f i}^{(5)}(\xi)+(N-1)^{-1} \sum_{\xi}^{N} \sum_{\xi^{\prime}}^{N-1} M_{f i}^{(5)}\left(\xi, \xi^{\prime}\right)+\ldots
$$

where the matrix element $M_{f i}$ is given by

$$
\begin{gathered}
M_{f i}=\sum_{s=0}\left\langle f\left|\left(H_{\mathrm{int}} T_{0}\right)^{s} H_{\mathrm{int}}\right| i\right\rangle ; \\
T_{0} \approx\left(E_{i}-H_{0}\right)^{-1} .
\end{gathered}
$$

In (4), the first term corresponds to interactions of the type [AAA], whilst the third term correctly accounts for interactions that take place across two optical centers [AAABB]. The second term relates to processes [AAAAA] which involve no delocalized couplings; it accounts for an optical Kerr effect shift in energy levels, and since it has no influence on issues of non-locality, it is of no further interest to us here. 


\section{LOCALIZED MECHANISM}

To study quantitatively the non-localised contributions to the two nonlinear optical processes in this paper, it is essential to calculate the rates of the leading order localised processes. In order to write such quantum amplitudes, is it necessary to construct the radiation tensors and material tensors, of which both are dependent on one another: a material response is frequency-dependent, whilst the properties of the radiation within a medium depends on the nature of its constituents.

The position-dependent radiation tensors for SHG and SPDC, respectively, are;

$$
\begin{aligned}
\rho_{i j k}^{S H G} & =\left\langle\left(q^{\prime}+1\right)\left(\boldsymbol{k}^{\prime}, \eta^{\prime}\right)\left|d_{i}^{\perp}\right| q^{\prime}\left(\boldsymbol{k}^{\prime}, \eta^{\prime}\right)\right\rangle\left\langle(q-2)(\boldsymbol{k}, \eta)\left|d_{j}^{\perp}\right|(q-1)(\boldsymbol{k}, \eta)\right\rangle \\
& \times\left\langle(q-1)(\boldsymbol{k}, \eta)\left|d_{k}^{\perp}\right| q(\boldsymbol{k}, \eta)\right\rangle, \\
\rho_{i j k}^{S P D C}= & \left\langle\left(q^{\prime}+2\right)\left(\boldsymbol{k}^{\prime}, \eta\right)\left|d_{i}^{\perp}\right|\left(q^{\prime}+1\right)\left(\boldsymbol{k}^{\prime}, \eta\right)\right\rangle\left\langle\left(q^{\prime}+1\right)\left(\boldsymbol{k}^{\prime}, \eta\right)\left|d_{j}^{\perp}\right| q^{\prime}\left(\boldsymbol{k}^{\prime}, \eta\right)\right\rangle \\
& \times\left\langle(q-1)(\boldsymbol{k}, \eta)\left|d_{k}^{\perp}\right| q(\boldsymbol{k}, \eta)\right\rangle .
\end{aligned}
$$

Which, with the aid of (3), the above radiation tensors can be expanded to become:

$$
\begin{aligned}
& \rho_{i j k}^{S H G}=i\left(\frac{\hbar \varepsilon_{0} \omega}{2 V n^{2}}\right)^{3 / 2}\left(\frac{n^{2}+2}{3}\right)^{3}\left\{\left(q^{\prime}+1\right)(q-1) q\right\}^{1 / 2} \vec{e}_{i} e_{j} e_{k}, \\
& \rho_{i j k}^{S P D C}=-i\left(\frac{\hbar \varepsilon_{0} \omega}{2 V n^{2}}\right)^{3 / 2}\left(\frac{n^{2}+2}{3}\right)^{3}\left\{\left(q^{\prime}+1\right)\left(q^{\prime}+2\right) q\right\}^{1 / 2} e_{i} \vec{e}_{j} \vec{e}_{k} .
\end{aligned}
$$

To go from the radiation tensor to the matrix element, we need to account for the coupling with the matter, which we can do by application of the correct material tensor. In non-relativistic quantum electrodynamics it is convenient to construct the material response in terms of a molecular tensor. These molecular tensors are easily applicable to bulk material in the form of susceptibilities ${ }^{1-2}$, connected as follows:

$$
\begin{aligned}
\chi_{i(j k)}^{(2)}(\mp \omega, \mp \omega ; \pm 2 \omega)= & \frac{N^{\prime}}{2 \varepsilon_{0}}\left(\frac{n^{2}+2}{3}\right)^{3} \sum_{r, s}\left[\frac{\mu_{i}^{0 r} \mu_{j}^{r s} \mu_{k}^{s 0}}{\left(E_{s 0}-\hbar \omega\right)\left(E_{r 0}-2 \hbar \omega\right)}\right. \\
& \left.+\frac{\mu_{j}^{0 r} \mu_{i}^{r s} \mu_{k}^{s 0}}{\left(E_{s 0}-\hbar \omega\right)\left(E_{r 0}+\hbar \omega\right)}+\frac{\mu_{j}^{0 r} \mu_{k}^{r s} \mu_{i}^{s 0}}{\left(E_{s 0}+2 \hbar \omega\right)\left(E_{r 0}+\hbar \omega\right)}+\{j \leftrightarrow k\}\right] \\
& =\frac{N^{\prime}}{\varepsilon_{0}}\left(\frac{n^{2}+2}{3}\right)^{3} \beta_{i(j k)}(\mp \omega, \mp \omega ; \pm 2 \omega),
\end{aligned}
$$

where $\chi_{i(j k)}^{(2)}$ is the frequency-dependent nonlinear-susceptibility tensor, $\beta_{i(j k)}$ is the molecular hyperpolarizability tensor, $N^{\prime}$ is the number density of active centers, equivalent to $V^{-1}$, and $\{j \leftrightarrow k\}$ signifies the other terms by exchanging these indices. Taking all of the above into consideration, we can write the total quantum amplitude for both SHG and SPDC for the localized [AAA] coupling as: 


$$
M_{f i}^{S H G}=-\frac{i}{2}\left(\frac{\hbar \varepsilon_{0} \omega}{2 V n^{2}}\right)^{3 / 2}\left(\frac{n^{2}+2}{3}\right)^{3}\left\{\left(q^{\prime}+1\right)(q-1) q\right\}^{1 / 2} \vec{e}_{i} e_{j} e_{k} \chi_{i(j k)}^{(2)}(\omega, \omega ;-2 \omega),
$$

and we also have;

$$
M_{f i}^{S P D C}=2^{-5 / 2} i\left(\frac{\hbar \varepsilon_{0} \omega}{2 V n^{2}}\right)^{3 / 2}\left(\frac{n^{2}+2}{3}\right)^{3}\left\{\left(q^{\prime}+1\right)\left(q^{\prime}+2\right) q\right\}^{1 / 2} e_{i} \vec{e}_{j}^{\prime} \vec{e}_{k}^{\prime} \chi_{i(j k)}^{(2)}(-\omega,-\omega ; 2 \omega) .
$$

\section{DELOCALIZED MECHANISM}

Delocalized contributions to the overall rate of down-conversion and harmonic generation entail a secondary mechanism involving the coupling of any optical center A with a new point in the optical medium, which we label B. No constraints are imposed on the relative positions of A and B. We assume that A and B are electronically equivalent, so that the labelling of $\mathrm{A}$ and $\mathrm{B}$ is arbitrary - the former being associated only with the position of input photon annihilation. For this reason there is no need to entertain role reversal $\mathrm{A} \leftrightarrow \mathrm{B}$. Through non-local interactions the correlated photon pair thus emerges from two, delocalized points within the medium, whilst the harmonic is produced from two input photons that were absorbed at completely separated points within the non-linear crystal.

The delocalized mechanisms entails five photon-matter interaction events, so we now have recourse to (4) for the corresponding quantum amplitude:

$$
\begin{aligned}
M_{A B}^{S H G} & =-\frac{i}{2 N^{\prime}}\left(\frac{\hbar \omega}{n^{2}}\right)^{\frac{3}{2}}\left(\frac{\left\{\left(q^{\prime}+1\right)(q-1) q\right\} \varepsilon_{0}}{V}\right)^{\frac{1}{2}} \frac{1}{n^{2}} \mathrm{e}^{\mathrm{i} k^{\prime} \cdot r_{\mathrm{B}}} \\
& \times \vec{e}_{i}^{\prime} e_{k} e_{m} \chi_{i(j k)}^{(2)}(+\omega,+\omega ;-2 \omega) \chi_{l m}^{(1)}(-\omega ; \omega) V_{j l}\left(n, k^{\prime}, \boldsymbol{r}_{\mathrm{B}}\right),
\end{aligned}
$$

and

$$
\begin{aligned}
M_{A B}^{S P D C} & =2^{-5 / 2} \frac{i}{N^{\prime}}\left(\frac{\hbar \omega}{n^{2}}\right)^{\frac{3}{2}}\left(\frac{\left\{\left(q^{\prime}+1\right)\left(q^{\prime}+2\right) q\right\} \varepsilon_{0}}{V}\right)^{\frac{1}{2}} \frac{1}{n^{2}} \mathrm{e}^{-\mathrm{i} k^{\prime} \cdot r_{\mathrm{B}}} \\
& \times e_{i} \vec{e}_{k}^{\prime} \vec{e}_{m}^{\prime} \chi_{i(j k)}^{(2)}(-\omega,-\omega ; 2 \omega) \chi_{l m}^{(1)}(-\omega ; \omega) V_{j l}\left(n, k^{\prime}, \boldsymbol{r}_{\mathrm{B}}\right),
\end{aligned}
$$

where $\chi_{l m}^{(1)}$ is the conventional linear susceptibility tensor, which is related to the molecular polarizability $\alpha$, as given below

$$
\begin{aligned}
\chi_{l m}^{(1)}(-\omega, \omega) & =\frac{N^{\prime}}{\varepsilon_{0}}\left(\frac{n^{2}+2}{3}\right)^{2} \sum_{r}\left[\frac{\mu_{l}^{0 r} \mu_{m}^{r 0}}{E_{r 0}^{\mathrm{B}}+\hbar \omega}+\frac{\mu_{m}^{0 r} \mu_{l}^{r 0}}{E_{r 0}^{\mathrm{B}}-\hbar \omega}\right] \\
& =\frac{N^{\prime}}{\varepsilon_{0}}\left(\frac{n^{2}+2}{3}\right)^{2} \alpha_{l m}(-\omega, \omega)
\end{aligned}
$$

and $V_{j l}\left(n, k^{\prime}, \boldsymbol{r}_{\mathrm{B}}\right)$ is the retarded resonance electric dipole coupling; ${ }^{38}$ 


$$
V_{j l}\left(n, k^{\prime}, \boldsymbol{r}_{\mathrm{B}}\right)=\frac{\mathrm{e}^{i n k^{\prime} r_{\mathrm{B}}}}{4 \pi \varepsilon_{0} r_{\mathrm{B}}^{3}}\left[\left(\delta_{j l}-3 \hat{r}_{\mathrm{B} j} \hat{r}_{\mathrm{B} l}\right)\left(1-i n k^{\prime} r_{\mathrm{B}}\right)-\left(\delta_{j l}-\hat{r}_{\mathrm{B} j} \hat{r}_{\mathrm{B} l}\right) n^{2} k^{\prime 2} r_{\mathrm{B}}^{2}\right],
$$

in which $n$ is the complex refractive index. Fully engaging the complex value in this formulation enables dissipative, line-shape related effects to be incorporated in the theory, without contriving an additional damping factor.

\section{SYMMETRY CONSIDERATIONS}

Previous work by the authors using rigorous computational methods has highlighted an indicative figure of a $2 \%$ enhancement to the rate of SPDC from the delocalized mechanism. ${ }^{23}$ Within the simplifications outlined earlier, in Section 2, we now consider the possible enhancement to SHG, paying particular heed to the time-reversal symmetry that links SHG to SPDC. As is well known, the fundamental symmetries of significance to optical and electromagnetic phenomena are the parities with respect to charge, space and time inversion - operations denoted by $C, \mathcal{P}$, and $\mathcal{T}$ respectively. ${ }^{39}$ All electromagnetic interactions exhibit $C \mathcal{P} \mathcal{T}$ symmetry, though since charge conjugation is of no interest it suffices in optics generally to consider $\mathcal{P} \mathcal{T}$ symmetry alone. Since each electric field vector is odd in character with respect to spatial inversion, and so too is each electric transition dipole moment, it is evident that the matrix elements for SHG and SPDC are both of even parity overall; the same is more obviously true for their parity in time. Moreover, it is relatively easy to show that the retarded resonance coupling tensor (18) is even in character with respect to inversion time and in space - under conditions where damping and dissipation effects can be neglected. The relationship between SHG and SPDC is that one is fundamentally the time-inverse of the other. Here, it is necessary to be clear about what 'timeinverse' signifies; here it is used in the sense of an ' $H T$ ' operation, combining the reversal of explicit time variables with Hermitian conjugation, which also subsumes complex variable conjugation, ${ }^{40,1}$ this is how it operates on the sequence and temporal sense of the Feynman diagrams that are commonly used to identify the form of the molecular response tensors. It then follows that in the rate equations, where the full matrix element (including both local and delocalized parts) is taken in a product with its complex conjugate, theory delivers a result that has exactly the same form for both SHG and SPDC - subject to factors connected with the input mode intensities. Of course, SHG has a nonlinear (quadratic) dependence on the input irradiance, whereas SPDC has a strictly linear dependence.

\section{PARTICLE SIZE EFFECTS}

The broad physical consequences of pairwise coupling, in connection with SPDC in bulk media, has already been established in recent work. ${ }^{22-23}$ Here, we focus on another aspect, concerned with the extent of delocalization. In a system of nanoparticles, within each of which there will generally be a system of optical centers able to play the role of one of a coupled pair, it is interesting to observe the effect of increasing particle size. Through detailed computational modelling it has been found that there is a highly marked variation in frequency conversion efficiency; in fact this applies to both SPDC and SHG. Aside from local statistical fluctuations there is a sharp rise in efficiency extending to about half of the longer of the two (input and output) wavelengths - beyond which, a decline occurs leading to eventual convergence on a rate that is slightly enhanced, to an approximately $2 \%$ increase in a typical case. This signifies that the conversion efficiency increases up to a limit that represents an optimum size, at which an enhancement of around $40 \%$ can be identified. This trend is summarized in the Table below, where results are reported for calculations based on the parameter values given in the caption. A key finding is that the propensity for either form of optical nonlinearity can prove markedly larger per unit volume than for a bulk of the same material, suggesting a significant design criterion. 


\begin{tabular}{|c|c|c|c|c|c|c|c|c|c|}
\hline Radius $/ \lambda$ & $<0.2$ & 1.0 & 1.33 & 2.0 & 3.0 & 4.0 & 5.0 & 6.0 & $\infty$ \\
\hline Enhancement $\%$ & 0 & +33 & +40 & +15 & +3 & +6 & +4 & +2 & +2 \\
\hline
\end{tabular}

Table 1. Results of model calculation on the extent of conversion efficiency enhancement in nanoparticles of dimensions comparative to the wavelength of input for SHG, or the output for SPDC. Values for the material parameters are chosen to approximately match beta-barium borate: $n \cong 1.7+0.1$; and $\chi_{x(x x)}^{(2)}=5.8, \chi_{z(x x)}^{(2)}=0.29$ (manufacturer reported values relative to potassium dihydrogen phosphate standard)

\section{DISCUSSION}

A delocalized mechanism for nonlinear optical processes, exhibiting a new form of quantum uncertainty, has been highlighted for the specific cases of spontaneous parametric down conversion and second harmonic generation. The introduction of the vacuum field as a means for energy transfer between separate optical interaction sites, extending beyond the usual constraints of the uncertainty principle, has led to new physics in parametric frequency conversion.

The resulting nonlocal effects in SPDC are expected to be detectable via the temporal and lateral broadening of the photonic output. The latter manifestation of the delocalized mechanism signifies that in applications such as 'ghost imaging ${ }^{41-44}$, the resolution of the image will be to some extent jeopardised by an insurmountable phenomenon of quantum origin - notwithstanding the experimental limitations when dealing with physical optics ${ }^{45}$ - as perfect imaging would require each correlated pair to originate from the same spatial origin. On a similar note, two photons arriving at distinct and well-separated locations producing the output harmonic in SHG can be anticipated to have implications on the resolution of SHG microscopy: perfect imaging would require each photon to annihilate at precisely the same centre.

As we have shown, the remarked enhancement of conversion efficiency, exhibited in the calculated results, indicates a factor that should play into the design criteria for nanoparticle composite nonlinear optical materials.

*david.andrews@physics.org

\section{ACKNOWLEDGMENTS}

We gratefully acknowledge computational assistance from Dr Garth A. Jones.

\section{REFERENCES}

[1] Andrews, D. L. and Allcock, P., [Optical Harmonics in Molecular Systems], Wiley-VCH, Weinheim (2002).

[2] Boyd, R. W., [Nonlinear Optics], Academic Press, (2003).

[3] Sutherland, R. L., [Handbook of nonlinear optics], CRC press, (2003).

[4] Campagnola, P. J. and Dong, C. Y., "Second harmonic generation microscopy: principles and applications to disease diagnosis," Laser \& Photon. Rev. 5, 13-26 (2011).

[5] Chen, X., Nadiarynkh, O., Plotnikov, S. and Campagnola, P. J., "Second harmonic generation microscopy for quantitative analysis of collagen fibrillar structure," Nature protocols 7, 654-669

(2012). 
[6] Cicchi, R., Vogler, N., Kapsokalyvas, D., Dietzek, B., Popp, J. and Pavone, F. S., "From molecular structure to tissue architecture: collagen organization probed by SHG microscopy," Journal of biophotonics 6, 129-142 (2013).

[7] Vielreicher, M., Schürmann, S., Detsch, R., Schmidt, M., Buttgereit, A., Boccaccini, A. and Friedrich, O., "Taking a deep look: modern microscopy technologies to optimize the design and functionality of biocompatible scaffolds for tissue engineering in regenerative medicine," Journal of the Royal Society Interface 10, 20130263 (2013).

[8] Bancelin, S., Aimé, C., Gusachenko, I., Kowalczuk, L., Latour, G., Coradin, T. and Schanne-Klein, M.C., "Determination of collagen fibril size via absolute measurements of second-harmonic generation signals," Nat. Commun. 5, (2014).

[9] Shen, Y., "Surface properties probed by second-harmonic and sum-frequency generation," Nature 337, 519-525 (1989).

[10] Corn, R. M. and Higgins, D. A., "Optical second harmonic generation as a probe of surface chemistry," Chem. Rev. 94, 107-125 (1994).

[11] Wu, H., Yu, H., Yang, Z., Hou, X., Su, X., Pan, S., Poeppelmeier, K. R. and Rondinelli, J. M., "Designing a deep-ultraviolet nonlinear optical material with a large second harmonic generation response," J. Am. Chem. Soc. 135, 4215-4218 (2013).

[12] Liu, S., Sinclair, M. B., Saravi, S., Keeler, G. A., Yang, Y., Reno, J., Peake, G. M., Setzpfandt, F., Staude, I. and Pertsch, T., "Resonantly enhanced second-harmonic generation using III-V semiconductor all-dielectric metasurfaces," Nano Lett. 16, 5426-5432 (2016).

[13] Seyler, K. L., Schaibley, J. R., Gong, P., Rivera, P., Jones, A. M., Wu, S., Yan, J., Mandrus, D. G., Yao, W. and Xu, X., "Electrical control of second-harmonic generation in a WSe2 monolayer transistor," Nat. Nanotechnol. 10, 407-411 (2015).

[14] Malard, L. M., Alencar, T. V., Barboza, A. P. M., Mak, K. F. and de Paula, A. M., “Observation of intense second harmonic generation from MoS 2 atomic crystals," Phys. Rev. B 87, 201401 (2013).

[15] Horodecki, R., Horodecki, P., Horodecki, M. and Horodecki, K., “Quantum entanglement," Rev. Mod. Phys. 81, 865-942 (2009).

[16] Simon, D. S., Jaeger, G. and Sergienko, A. V., [Quantum Metrology, Imaging, and Communication], Springer, (2017).

[17] Müller, M., Bounouar, S., Jöns, K. D., Glässl, M. and Michler, P., “On-demand generation of indistinguishable polarization-entangled photon pairs," Nat. Photonics 8, 224-228 (2014).

[18] Wang, X.-L., Chen, L.-K., Li, W., Huang, H.-L., Liu, C., Chen, C., Luo, Y.-H., Su, Z.-E., Wu, D. and Li, Z.-D., "Experimental ten-photon entanglement," Phys. Rev. Lett. 117, 210502 (2016).

[19] Nielsen, M. A. and Chuang, I., [Quantum computation and quantum information], AAPT, (2002).

[20] Wilde, M. M., [Quantum information theory], Cambridge University Press, (2013).

[21] Caspani, L., Reimer, C., Kues, M., Roztocki, P., Clerici, M., Wetzel, B., Jestin, Y., Ferrera, M., Peccianti, M. and Pasquazi, A., "Multifrequency sources of quantum correlated photon pairs on-chip: a path toward integrated Quantum Frequency Combs," Nanophoton. 5, 351-362 (2016).

[22] Forbes, K. A., Ford, J. S. and Andrews, D. L., "Nonlocalized generation of correlated photon pairs in degenerate down-conversion," Phys. Rev. Lett. 118, 133602 (2017).

[23] Forbes, K. A., Ford, J. S., Jones, G. A. and Andrews, D. L., "Quantum delocalisation in photon-pair generation," Manuscript submitted for publication, (2017).

[24] Loudon, R., [The Quantum Theory of Light], Oxford University Press, Oxford (2000).

[25] Grynberg, G., Aspect, A. and Fabre, C., [Introduction to Quantum Optics: From the Semi-Classical Approach to Quantized Light], Cambridge University Press, Cambridge (2010).

[26] Craig, D. P. and Thirunamachandran, T., [Molecular Quantum Electrodynamics: An Introduction to Radiation-Molecule Interactions], Dover Publications, Mineola, NY (1998).

[27] Power, E. A. and Zienau, S., "Coulomb gauge in non-relativistic quantum electro-dynamics and the shape of spectral lines," Philos. Trans. R. Soc. A 251, 427-454 (1959).

[28] Woolley, R., "Molecular quantum electrodynamics,” Proc. R. Soc. A 321, 557-572 (1971).

[29] Power, E. A. and Thirunamachandran, T., "The multipolar Hamiltonian in radiation theory," Proc. R. Soc. A 372, 265-273 (1980).

[30] Bradshaw, D. S. and Andrews, D. L., "Manipulating particles with light: radiation and gradient forces," Eur. J. Phys. 38, 034008 (2017). 
[31] Bradshaw, D. S., Forbes, K. A., Leeder, J. M. and Andrews, D. L., "Chirality in optical trapping and optical binding," Photonics 2, 483-497 (2015).

[32] Chen, H., Jiang, Y., Wang, N., Lu, W., Liu, S. and Lin, Z., "Lateral optical force on paired chiral nanoparticles in linearly polarized plane waves," Opt. Lett. 40, 5530-5533 (2015).

[33] Forbes, K. A. and Andrews, D. L., "Chiral discrimination in optical binding," Phys. Rev. A 91, 053824 (2015).

[34] Williams, M. D., Ford, J. S. and Andrews, D. L., "Hyper-Rayleigh scattering in centrosymmetric systems," J. Chem. Phys. 143, 124301 (2015).

[35] Forbes, K. A., Bradshaw, D. S. and Andrews, D. L., "Identifying diamagnetic interactions in scattering and nonlinear optics," Phys. Rev. A 94, 033837 (2016).

[36] Juzeliūnas., G., "Microscopic theory of quantization of radiation in molecular dielectrics: Normalmode representation of operators for local and averaged (macroscopic) fields," Phys. Rev. A 53, 35433558 (1996).

[37] Juzeliūnas., G., "Microscopic theory of quantization of radiation in molecular dielectrics. II. Analysis of microscopic field operators," Phys. Rev. A 55, 929-934 (1997).

[38] Juzeliūnas., G. and Andrews, D. L., "Quantum electrodynamics of resonance energy transfer," Adv. Chem. Phys. 112, 357-410 (2000).

[39] Greenberg, O., "Why is CPT Fundamental?," Foundations of Physics 36, 1535-1553 (2006).

[40] Stedman, G. E., [Diagram Techniques in Group Theory], Cambridge University Press, Cambridge, U.K. (1990).

[41] Pittman, T., Shih, Y., Strekalov, D. and Sergienko, A., "Optical imaging by means of two-photon quantum entanglement," Phys. Rev. A 52, R3429 (1995).

[42] Gatti, A., Brambilla, E., Bache, M. and Lugiato, L. A., "Ghost imaging with thermal light: comparing entanglement and classicalcorrelation," Phys. Rev. Lett. 93, 093602 (2004).

[43] Morris, P. A., Aspden, R. S., Bell, J. E., Boyd, R. W. and Padgett, M. J., "Imaging with a small number of photons," Nat. Commun. 6, (2015).

[44] Cheng, J., "Theory of ghost scattering with biphoton states," Photonics Research 5, $41-45$ (2017).

[45] Rubin, M. H. and Shih, Y., "Resolution of ghost imaging for nondegenerate spontaneous parametric down-conversion,” Phys. Rev. A 78, 033836 (2008). 